\title{
Avaliação da deformação miocárdica pela ecocardiografia feature tracking em gatos com defeito perimembranoso do septo ventricular
}

\author{
[Evaluation of myocardial deformation by the ecocardiography feature tracking in cats with \\ perimembranosous defect of the septo ventricular] \\ M.R. Coelho ${ }^{1}$, R.A.L. Muzzi $i^{2}$, E.M.S. Dorneles ${ }^{2}$, L.E.D. Oliveira ${ }^{1}$, \\ C.B. Abreu ${ }^{1}$, L.L.A. Furtado ${ }^{1}$, L.A.L. Muzzi ${ }^{2}$ \\ ${ }^{1}$ Aluno de pós-graduação - Universidade Federal de Lavras - Lavras, MG \\ ${ }^{2}$ Universidade Federal de Lavras - Lavras, MG
}

\begin{abstract}
RESUMO
O desenvolvimento de novas ferramentas, como a ecocardiografia bidimensional feature tracking (2DFTI), permite diagnosticar, de forma precoce, se há disfunção miocárdica em doenças cardíacas, inclusive as congênitas. O defeito septal ventricular (DSV) é a alteração congênita mais observada em felinos, no entanto pouco se sabe sobre a disfunção cardíaca nessa cardiopatia, especialmente em animais assintomáticos. O objetivo deste estudo foi avaliar, por meio do 2D-FTI, a deformação miocárdica ventricular esquerda pela mensuração dos índices ecocardiográficos strain $(\mathrm{St})$ e strain rate $(\mathrm{StR})$ radial, circunferencial e longitudinal, em gatos saudáveis e com DSV. Foram avaliados 12 gatos saudáveis e seis gatos com DSV para obtenção de St e StR em diversos segmentos miocárdicos. No sentido longitudinal, houve diferença estatística $(\mathrm{P}<0,05)$ para os segmentos septal basal, mediano e apical epicárdicos $(\mathrm{P}=0,0017 ; \mathrm{P}<0,0001 ; \mathrm{P}=0,0288)$, lateral mediano epicárdico $(\mathrm{P}=0,0327)$, septal mediano endocárdico $(\mathrm{P}=0,0035)$, lateral mediano endocárdico $(\mathrm{P}=0,0461)$, St epicárdico $(\mathrm{P}=0,0250)$ e St global $(\mathrm{P}=0,0382)$. Também houve diferença no segmento lateral mediano circunferencial endocárdico $(\mathrm{P}=0,0248)$, lateral mediano radial ( $\mathrm{St}: \mathrm{P}=0,0409$; StR: $\mathrm{P}=0,0166)$ e posterior mediano radial $(\mathrm{P}=0,0369)$. $\mathrm{O}$ estudo evidenciou que, mesmo em animais assintomáticos com DSV, há redução na deformação miocárdica ventricular principalmente no sentido longitudinal, demonstrando maior vulnerabilidade dessas fibras.
\end{abstract}

Palavras-chave: cardiopatia congênita, strain e strain rate ventricular, 2D-FTI

\begin{abstract}
The development of new tools, such as two-dimensional feature tracking (2D-FTI), allows early diagnosis of myocardial dysfunction in heart diseases including congenital heart disease. The ventricular septal defect (VSD) is the most frequently observed congenital abnormality in cats, however, little is known about cardiac dysfunction, especially in asymptomatic animals. The objective of this study was to evaluate the left ventricular myocardial deformation through 2D-FTI by the measurement of the radial, circumferential and longitudinal echocardiographic strain (St) and strain rate (StR) indices. Twelve healthy cats and six cats with VSD were evaluated to obtain St and StR in several myocardial segments. In the longitudinal direction, there was a statistical difference $(P<0.05)$ for the epimyocardial basal septal, mid-septal, apical septal $(P=0.0017 ; \quad P<0.0001 ; P=0.0288)$, epimyocardial mid-lateral $(P=0.0327)$, endomyocardial mid-septal $(P=0.0035)$, endomyocardial mid-lateral $(P=0.0461)$, St epimyocardial $(P=0.0250)$ and St global $(P=0.0382)$. There was also difference in the circumferential endomyocardial mid-lateral segment $(P=0.0248)$, radial mid-lateral (St: $P=0.0409 ;$ StR: $P=0.0166)$ and radial mid-posterior $(P=0.0369)$. The study showed that even in asymptomatic animals with VSD there is a reduction in ventricular myocardial deformation mainly in the longitudinal direction, demonstrating the fragility of these fibers.
\end{abstract}

Keywords: congenital cardiopathy; ventricular strain e strain rate; $2 D-F T I$

Recebido em 19 de setembro de 2018

Aceito em 18 de setembro de 2019

E-mail: marianacoelhorc@gmail.com 


\section{INTRODUÇÃO}

Entre as cardiopatias congênitas em felinos, o defeito do septo ventricular (DSV) é a alteração mais observada na espécie (Tidholm et al., 2015). Compreende um orifício no septo interventricular que permite a passagem de sangue de um ventrículo ao outro (Roland, 2011). Esse defeito no septo pode ser classificado, de acordo com sua localização anatômica, em perimembranoso, supracristal, muscular ou posterior. Em pequenos animais, os defeitos do septo interventricular perimembranosos são os mais observados, e os defeitos musculares são incomuns (Bussadori e Pradelli, 2015).

Os achados clínicos do DSV são variáveis e dependentes da dimensão do defeito e das pressões ventriculares, que irão influenciar o grau e a direção do desvio sanguíneo. Em pacientes que apresentam um DSV com pequeno diâmetro, pode ser observado sopro sistólico alto e ausência de sinais radiográficos, sem repercussão hemodinâmica (Kittleson e Kienle, 1998; Macdonald, 2006). Considerando que a pressão do ventrículo esquerdo é superior à do ventrículo direito, em condições cardíacas normais, ocorre desvio do fluxo sanguíneo da esquerda para a direita, havendo sobrecarga de átrio e ventrículo esquerdos em decorrência do aumento do retorno sanguíneo pela veia pulmonar. O diâmetro do DSV é determinado pela comparação com o diâmetro da aorta, em que DSVs pequenos equivalem a valores menores que $50 \%$ do diâmetro da aorta, e DSVs moderados ou graves a valores maiores que $50 \%$ (Boon, 2011). Quando o diâmetro do defeito é maior, alguns sinais de policitemia e insuficiência cardíaca congestiva (ICC) esquerda ou direita podem ser observados (Ware, 2015).

O diagnóstico geralmente é confirmado por meio do exame ecocardiográfico, que demonstra dilatações ventricular e atrial esquerdas para os defeitos moderados a grandes (Strickland, 2008). Além disso, a ecocardiografia permite identificar e definir o tamanho do defeito, determinar a direção do fluxo sanguíneo, avaliar suas consequências hemodinâmicas e remodelamento ventricular, quantificando o desvio (Bulmer, 2011; Bussadori e Pradelli, 2015).
O tratamento é variável conforme a gravidade do quadro clínico do paciente, sendo que animais com defeitos pequenos e sem remodelamento cardíaco não devem ser tratados. Quando há remodelamento, o uso de inibidores da enzima conversora da angiotensina (ECA) pode ser benéfico (Pereira e Larsson, 2015). As opções terapêuticas para paciente com DSV incluem a terapia convencional para ICC esquerda, incluindo diuréticos, inibidores da ECA e inotrópicos positivos (Bulmer, 2011; Pereira e Larsson, 2015; Ware, 2015). O uso de agente redutores de pós-carga não é indicado para pacientes com hipertensão pulmonar importante, pois podem facilitar a reversão do desvio (Pereira e Larsson, 2015). O prognóstico é favorável quando o animal permanece assintomático (Côté et al., 2011).

Em medicina veterinária, a ferramenta ecocardiográfica feature tracking bidimensional (2D-FTI) tem sido utilizada em cães, para se obterem os índices ecocardiográficos strain $(\mathrm{St})$ e strain rate ( $\mathrm{StR})$, tanto em animais saudáveis, para se estabelecerem valores de referência e se padronizar a técnica (Chetboul et al., 2008; Wess et al., 2011), quanto em animais cardiopatas (Mantovani et al., 2015). No entanto, há pouca informação na literatura sobre o uso dessa ferramenta em gatos domésticos (Riesen et al., 2011), assim como valores de referência para St e StR longitudinal, radial ou circunferencial.

Assim, o objetivo deste trabalho foi avaliar a deformação miocárdica pelos índices St e StR radial, circunferencial e longitudinal em gatos com defeito perimembranoso do septo ventricular, sendo observado se há disfunção miocárdica.

\section{MATERIAL E MÉTODOS}

Os dados submetidos à análise foram obtidos do serviço de cardiologia do hospital veterinário da instituição. $\mathrm{O}$ estudo teve aprovação da Comissão de Ética no Uso de Animais (Ceua/Ufla), protocolo 052/11. Todos os pacientes atendidos foram submetidos a exame físico detalhado, eletrocardiograma e ecocardiograma. Os animais incluídos no estudo foram aqueles que apresentaram apenas a presença de DSV (Fig. 1), sem outras cardiopatias congênitas concomitantes, bem como ausência de afecções sistêmicas. 

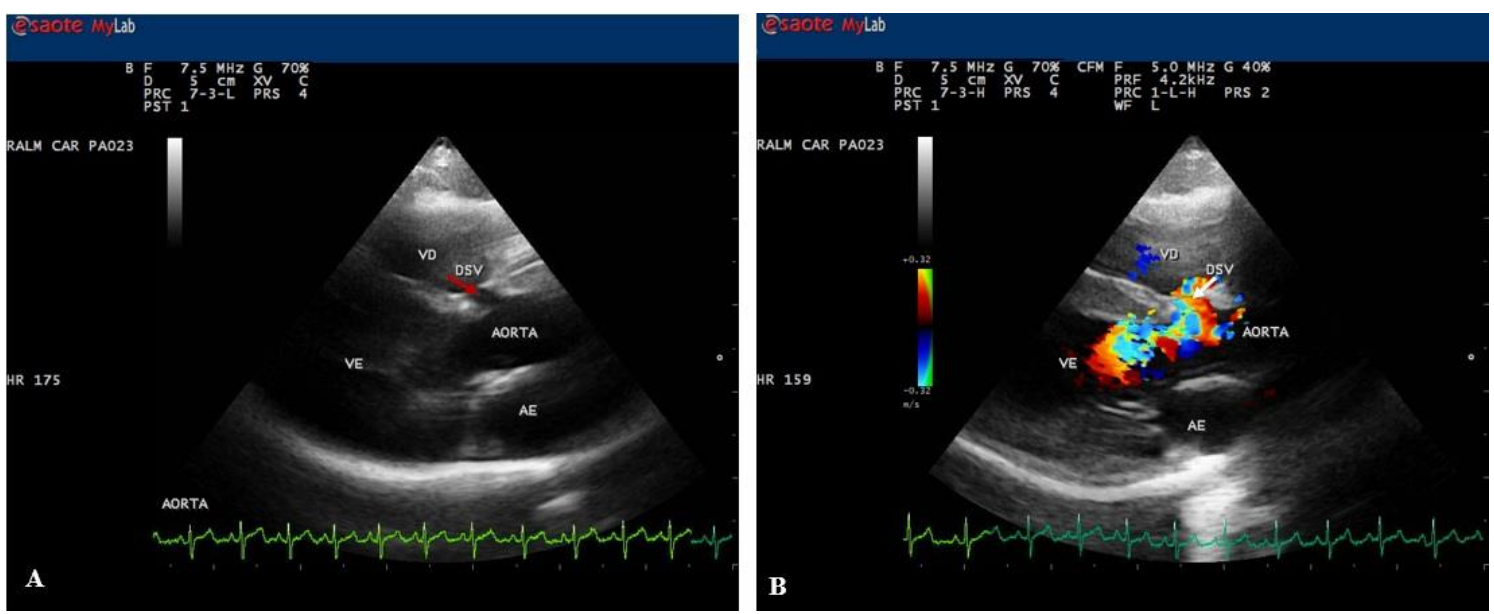

Figura 1. Imagens ecocardiográficas de um gato da raça Persa, um ano de idade e 4,5kg de peso. A - vista longitudinal cinco câmaras, modo bidimensional, demonstrando a presença de um defeito do septo ventricular (DSV) perimembranoso (seta vermelha). B - vista longitudinal cinco câmaras, modo Doppler em cores, demonstrando o trajeto do fluxo sanguíneo da esquerda para a direita, por meio do DSV perimembranoso (seta branca). Fonte: Serviço de Cardiologia do HV/Ufla.

Foram avaliados 18 animais, sendo o grupo controle composto por 12 gatos e o grupo com DSV composto por 6 gatos assintomáticos. Para a realização do exame ecocardiográfico (aparelho MyLab 40 Esaote $^{\circledR}$ ), os animais foram posicionados em decúbito lateral direito e esquerdo, por meio de contenção manual, com acompanhamento eletrocardiográfico no monitor. Para a ecocardiografia 2D-FTI, três a cinco ciclos cardíacos consecutivos utilizando monitorização contínua do ECG, com taxa de amostragem entre 50 e 100 frames/s, foram armazenados em formato digital para análise offline, utilizando-se o software XStrain, algoritmo optical flow (versão 10.1, Esaote). Os índices que representam a taxa e a velocidade de deformação miocárdica ventricular $(\mathrm{St}$ e $\mathrm{StR}$, respectivamente) foram obtidos usando-se o método 2D-FTI, de acordo com Carnabuci et al. (2013). Assim, para se obter o St e o StR longitudinal, foi utilizado o corte apical quatro câmaras esquerdo e, para os índices St e StR radial e circunferencial, foi utilizado o corte paraesternal direito transversal, no plano dos músculos papilares do ventrículo esquerdo (VE). Foram realizadas três medidas para cada variável para se obter a média dos seis segmentos avaliados nos três sentidos (radial, circunferencial e longitudinal).
A análise estatística foi realizada utilizando-se o software Graph Pad Prism ${ }^{\circledR}$ - version 5.0. Empregou-se o teste $\mathrm{t}$ de Student não pareado para comparação dos dados obtidos. Um valor de $\mathrm{P}<0,05$ foi usado para definir a significância estatística (Sampaio, 2002).

\section{RESULTADOS}

Todos os gatos avaliados eram do sexo masculino. No grupo controle, as raças encontradas foram sem padrão racial definido SRD (10), Persa (1) e Siamês (1); já no grupo de DSV, as raças encontradas foram Persa (4) e SRD (2). As características clínicas dos animais, bem como o diâmetro do defeito mensurado, estão apresentadas na Tab. 1 .

No exame ecocardiográfico convencional, todos os animais demonstraram dimensão de câmaras atrial e ventricular esquerdas dentro dos limites de normalidade. Em relação às câmaras direitas, também foi observada dimensão dentro dos limites de normalidade, uma vez que todos os animais apresentaram defeito de pequeno diâmetro. 


\section{Coelho et al.}

Tabela 1. Características clínicas e mensuração do defeito perimembranoso do septo ventricular dos animais avaliados apresentados como média e desvio-padrão.

\begin{tabular}{lcccccc} 
Animais & Idade (meses) & Peso em kg & $\begin{array}{c}\text { FC (batimentos } \\
\text { por minuto) }\end{array}$ & $\begin{array}{c}\text { Diâmetro } \\
\text { médio do } \\
\text { defeito em mm } \\
\text { (DSV) }\end{array}$ & $\begin{array}{c}\text { Diâmetro } \\
\text { médio da } \\
\text { aorta em mm } \\
\text { (Ao) }\end{array}$ & DSV/Ao \\
\hline $\begin{array}{l}\text { Grupo } \\
\text { controle } \\
(\mathrm{n}=12)\end{array}$ & $30,58 \pm 19,32$ & $4,20 \pm 0,80$ & $154,92 \pm 26,52$ & - & - & - \\
$\begin{array}{l}\text { Grupo DSV } \\
(\mathrm{n}=6)\end{array}$ & $36,00 \pm 26,29$ & $3,68 \pm 0,57$ & $188,67 \pm 27,24$ & $1,58 \pm 0,79$ & $6,18 \pm 0,6$ & 0,26 \\
\hline
\end{tabular}

FC: frequência cardíaca.

Para o índice St circunferencial, houve diferença significativa entre os grupos (controle $x$ DSV) para o segmento lateral mediano endocárdico $(-17,87 \pm 5,19 x-11,02 \pm 6,21 ; \mathrm{P}=0,0248)$. Não foi observada diferença estatística para os índices StR circunferencial e longitudinal. Já no sentido radial, observou-se diferença para os índices de St no segmento lateral $(25,72 \pm 10,96 \times 14,23 \pm 8,8$; $\mathrm{P}=0,0409)$, posterior mediano $(25,71 \pm 12,48 x$ $13,14 \pm 6,89 ; \mathrm{P}=0,0369)$, e $\mathrm{StR}$ no segmento lateral mediano $(2,60 \pm 0,88 \quad x \quad 1,57 \pm 0,41$; $\mathrm{P}=0,0166$ ).

No sentido longitudinal, para o St epicárdico, houve diferença para os segmentos septal basal
$(-22,86 \pm 7,93 x-10,11 \pm 2,91 ; \mathrm{P}=0,0017)$, septal mediano $(-17,29 \pm 4,11 x-7,28 \pm 0,80 ; \mathrm{P}<0,0001)$, apical $(-8,73 \pm 4,59 \quad x-4,08 \pm 1,26 ; \mathrm{P}=0,0288) \mathrm{e}$ lateral mediano $(-23,58 \pm 5,87 \quad x-13,58 \pm 12,56$; $\mathrm{P}=0,0327)$. Já para o $\mathrm{St}$ endocárdico, houve diferença nos segmentos septal mediano $(-14,10 \pm 4,16 x-7,77 \pm 2,39 ; \mathrm{P}=0,0035)$ e lateral mediano $(-18,44 \pm 5,94 \quad x \quad-11,14 \pm 8,28$, $\mathrm{P}=0,04461)$. Também houve diferença para o $\mathrm{St}$ epicárdico $\quad(-19,25 \pm 4,50 \quad x \quad-10,20 \pm 5,58$; $\mathrm{P}=0,0250) \quad$ e $\quad \mathrm{St}$ global $(-17,23 \pm 3,83$ $x-10,08 \pm 5,10 ; \mathrm{P}=0,0382)$. A Fig. 2 demonstra todos os segmentos miocárdicos que tiveram diferença significativa nos três sentidos avaliados (circunferencial, radial e longitudinal).
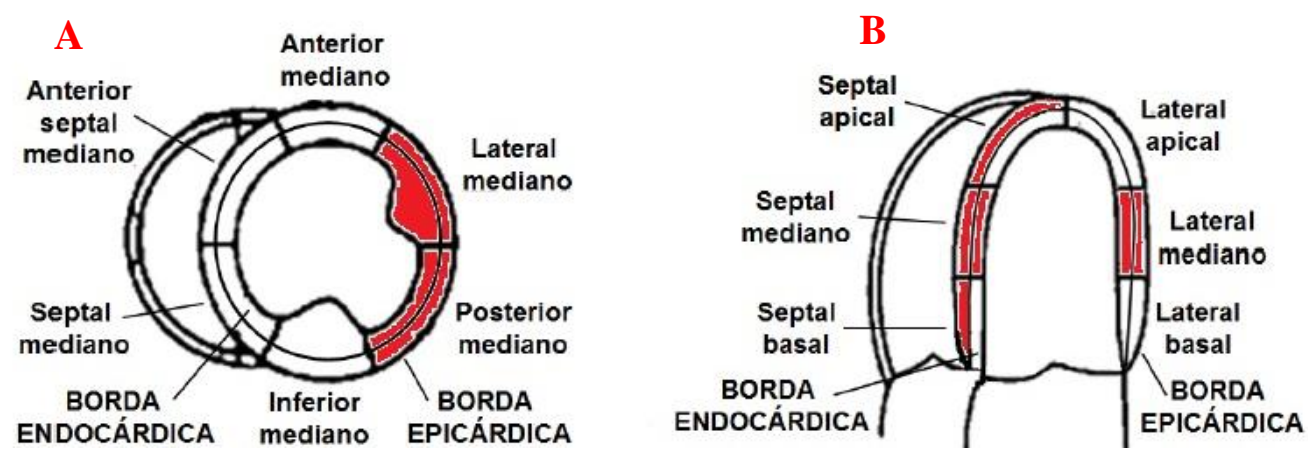

Figura 2. A. Imagem ilustrativa dos segmentos do ventrículo esquerdo (VE) em corte transversal no plano dos músculos papilares. As áreas em vermelho enfatizam as alterações regionais de strain (St) e strain rate (StR) radial e circunferencial em gatos com defeito do septo ventricular (DSV). B. Imagem ilustrativa dos segmentos do VE em corte apical quatro câmaras. As áreas em vermelho enfatizam as alterações regionais de St longitudinal em gatos com DSV.

\section{DISCUSSÃO}

O presente trabalho demonstrou que o uso da técnica 2D-FTI é viável para se obter a função miocárdica ventricular esquerda em gatos com DSV. A visão do corte apical quatro câmaras esquerdo demonstrou ser adequada para a obtenção das medidas St e StR longitudinais, apesar de a frequência cardíaca elevada resultar em uma menor resolução das imagens, dificultando a análise por essa técnica, como 
ocorreu também no estudo de Takano et al. (2015).

Os animais utilizados foram selecionados de forma aleatória de acordo com a rotina de cardiologia da instituição, o que explica a variação entre idade, raças e sexo dos animais. Dessa forma, apesar de ter sido observada uma prevalência da raça Persa para gatos com DSV, não há dados na literatura indicando essa raça como predisposta para a ocorrência do defeito (Côté et al., 2011).

Todos os gatos com defeito avaliados apresentaram um DSV de pequeno diâmetro, o que justifica eles serem assintomáticos (MacDonald, 2006), bem como não terem alterações significativas em nenhuma câmara cardíaca na ecocardiografia convencional. O caminho do fluxo sanguíneo no DSV se dá para o VE, ventrículo direito (VD), artéria pulmonar, pulmões, átrio esquerdo, e de volta ao VE. Apenas essas câmaras e vasos estarão apresentando sobrecarga de volume na presença de defeitos moderados a grandes. $\mathrm{O}$ átrio direito e a aorta não estão relacionados com o caminho desse desvio, então não apresentarão sobrecarga se não houver outro tipo de alteração envolvida (Boon, 2011).

Basicamente, a quantidade de sangue desviada do VE para o VD e, em seguida, para as artérias pulmonares, dependerá do tamanho do defeito, do gradiente de pressão entre as duas câmaras ventriculares e da pós-carga (Bussadori e Pradelli, 2015). A resistência ao fluxo em defeitos pequenos limita a quantidade de sangue desviado e seu impacto hemodinâmico, não ocorrendo alteração da função ventricular direita. Por outro lado, um defeito grande não cria resistência ao fluxo desviado, permitindo que uma grande quantidade de sangue passe do VE para o VD. Em alguns casos, o aumento da pressão pulmonar predispõe ao desenvolvimento de hipertensão arterial pulmonar e possível reversão do fluxo desviado, ou seja, da direita para a esquerda (síndrome de Eisenmenger) (Pereira e Larsson, 2015; Ware, 2015; Bussadori e Pradelli, 2015). No presente estudo, como os animais apresentavam apenas defeitos pequenos, não foi observada disfunção ventricular direita.

Este estudo possibilitou demonstrar alterações miocárdicas esquerdas regionais nos sentidos radial, circunferencial e longitudinal, sendo o segmento lateral mediano nos planos endocárdico e epicárdico o único a apresentar redução do strain nos três sentidos avaliados. O St no sentido longitudinal apresentou redução na deformação miocárdica tanto no plano endocárdico quanto no epicárdico, em diferentes segmentos, além de ter tido um valor de St global menor nos gatos com DSV. Em seres humanos com alterações cardíacas, as fibras no sentido longitudinal tendem a se deteriorar precocemente quando comparadas às do sentido circunferencial (Hung et al., 2010). No presente estudo, também pôde ser observado esse tipo de alteração, o que demonstra que a deformação miocárdica nessa região apresenta-se prejudicada mesmo no paciente assintomático. Isso provavelmente se deve à maior vulnerabilidade das fibras longitudinais do VE, sendo assim mais sensíveis quando há alterações miocárdicas (Zaky et al., 2014). Além disso, vários segmentos longitudinais apresentaram valores muito próximos da significância estatística, fato que demonstra uma tendência clara de que essas fibras se deterioram precocemente. Provavelmente, se o número de animais avaliados fosse maior, essas variáveis teriam valores significativos estatisticamente.

Também no sentido longitudinal, observou-se que os segmentos mais afetados são os que estão na região do septo interventricular (Fig. 1), o que condiz com a localização do defeito ser na região perimembranosa dos gatos avaliados. Nessa região, o músculo cardíaco é rico em fibras oblíquas e longitudinais que predominam na região média e apical do VE (Del Castillo, 2010).

Apesar de o sentido longitudinal ter demonstrado mais alteração na função ventricular, este estudo evidenciou que disfunções ventriculares locais podem ser detectadas mesmo nos sentidos radial e circunferencial, utilizando-se a ferramenta 2DFTI, sem preocupação com dependência de ângulo. $\mathrm{O}$ mesmo resultado foi observado por Takano et al. (2015) na avaliação de gatos saudáveis e gatos com cardiomiopatia hipertrófica utilizando-se a ferramenta speckle tracking (2D-STE). O fato de ter sido observada redução de St e StR na parede posterior do VE (segmentos lateral e posterior mediano) se deve ao conceito anatômico do coração ter formato helicoidal. O músculo cardíaco é formado por um único fascículo enrolado em si mesmo e 
ancorado nas suas extremidades, no anel pulmonar e no aórtico, com isso uma disfunção ventricular localizada pode ser detectada nos diferentes sentidos das fibras miocárdicas, como citado por Del Castillo (2010).

Os resultados encontrados demonstram a precocidade dessa análise, uma vez que nenhum dos gatos avaliados apresentava sintomatologia clínica de ICC direita ou esquerda nem remodelamento de câmaras cardíacas. Segundo Pereira e Larsson (2015), animais com defeitos pequenos e sem remodelamento não necessitam de tratamento. Porém, uma vez que foi diagnosticado um DSV, o animal deve ser avaliado periodicamente para monitoramento do estado clínico hemodinâmico do paciente. É importante também avaliar a função ventricular esquerda, pois essa é a primeira câmara a sofrer remodelamento cardíaco. Para isso, pode-se utilizar a ferramenta ecocardiográfica 2D-FTI, visto que o presente estudo demonstrou que essa técnica é aplicável para tal avaliação.

No entanto, este estudo apresenta algumas limitações. Primeiramente, trata-se de um estudo não invasivo, realizado em animais de companhia, sem dados hemodinâmicos obtidos de forma invasiva para corroborar o estado dos gatos avaliados. Além disso, existe uma alta dependência da qualidade de imagem para análise 2D-FTI, o que é difícil de se obter em gatos não sedados, uma vez que eles podem apresentar altas taxas de frequência cardíaca no exame ecocardiográfico.

Outra limitação deste estudo é o fato de que os dados obtidos podem não ser aplicáveis a todas as situações clínicas devido a diferenças de softwares, equipamentos, experiência do examinador e qualidade de imagem, o que pode influenciar os valores e a variabilidade dos dados. Além disso, são necessários mais estudos com a ecocardiografia 2D-FTI para se ter valores de referência em gatos saudáveis, bem como verificar se há uma influência de raça, gênero e idade sobre as variáveis 2D-FTI em gatos. Apesar das limitações, o estudo fornece uma referência preliminar da viabilidade dessa técnica em gatos saudáveis e em gatos com DSV, demonstrando a presença de uma disfunção miocárdica precoce que deve ser acompanhada nos pacientes para evitar progressões futuras.

\section{CONCLUSÃO}

A técnica 2D-FTI pode ser considerada uma ferramenta viável para a avaliação do miocárdio ventricular esquerdo pelos índices St e StR nos três sentidos analisados em gatos, especialmente aqueles com defeito septal ventricular. $\mathrm{O}$ estudo evidenciou que, mesmo em animais assintomáticos com defeito septal interventricular, há redução na deformação miocárdica ventricular principalmente no sentido longitudinal, o que demonstra maior vulnerabilidade dessas fibras.

\section{REFERÊNCIAS}

BOON, J.A. Evaluation of size, function, and hemodynamics. In: . (Eds.). Veterinary echocardiography. 2.ed. New Jersey: J. Willey, 2011. p.206-334.

BULMER, B.J. Sistema cardiovascular. In: (Eds.). Pediatria em pequenos animais. 1 ed. Rio de Janeiro: Elsevier, 2011. p.289-304.

BUSSADORI, C.; PRADELLI, D. Congenital cardiopathies. In: __ (Eds.). Clinical echocardiography of the dog and cat. 1 ed. St. Louis: Elsevier Masson, 2015. p.303-305.

CARNABUCI, C.; HANÅS, S.; LJUNGVALL, I. et al. Assessment of cardiac function using global and regional left ventricular endomyocardial and epimyocardial peak systolic strain and strain rate in healthy Labrador retriever dogs. Res. Vet. Sci., v.95, p.241-248, 2013.

CHETBOUL, V.; SERRES, F.; GOUNI, V. et al. Noninvasive assessment of systolic left ventricular torsion by 2-dimensional speckle tracking imaging in the awake dog: repeatability, reproducibility, and comparison with tissue Doppler imaging variables. J. Vet. Intern. Med., v.22, p.342-350, 2008 .

CÔTÉ, E.; MacDONALD, K.A.; MEURS, K.M.; SLEEPER, M.M. Congenital heart malformations. In: __ (Eds.). Feline Cardiology. 1 ed. New Jersey: J. Willey, 2011. p.91-92.

DEL CASTILLO, J.M. Strain Bidimensional ( $X$ Strain): utilização do método para avaliação de cardiopatias. Rev. Bras. Ecocardiogr., v.21, p.29-35, 2010. 
HUNG, C.L.; VERNA, A.; UNO, H. et al. Longitudinal and circumferential strain rate, left ventricular remodeling, and prognosis after myocardial infarction. J. Am. Coll. Cardiol., v.56, p.1812-1822, 2010.

KITTLESON, M.D.; KIENLE, R.D. Septal Defects. In: __. (Eds). Small animal cardiovascular medicine. 1 ed. St. Louis: Mosby Inc, 1998. p.233-238.

MACDONALD, K.A. Congenital heart diseases of puppies and kittens. Vet. Clin. N. Am. Small Anim. Pract., v.36, p.503-531, 2006.

MANTOVANI, M.M.; MUZZI, R.A.L.; PEREIRA, G.G. et al. Systolic cardiac function assessment by feature tracking echocardiography in dogs with myxomatous mitral valve disease. $J$. Small Anim. Pract., v.56, p.383-392, 2015.

PEREIRA， G.G.; LARSSON， M.H.M.A. Cardiopatias Congênitas em Cães e Gatos. In: . (Eds.). Tratado de Medicina Interna de Cães e Gatos. 1 ed. Rio de Janeiro: Roca, 2015. p.1119-1136.

ROLAND, R.M. Cardiopatia Congênita. In:___. (Eds.) Medicina interna felina. 6.ed. St. Louis: Saunders Elsevier, 2011, 425p.

SAMPAIO, I.B.M. Estatística aplicada à experimentação animal. 2.ed. Belo Horizonte: Fundação de Estudo e Pesquisa em Medicina Veterinária e Zootecnia 2002. 265p.
STRICKLAND, K.N. Congenital heart diseases. In: __. (Eds.) Manual of canine and feline cardiology. 4 ed. St. Louis: Saunders Elsevier, 2008. p.231-235.

TAKANO, H.; ISOGAI, T.; AOKI, T. et al. Feasibility of radial and circumferential strain analysis using 2D speckle tracking echocardiography in cats. J. Vet. Med. Sci., v.77, p.193-201, 2015.

TIDHOLM, A.; LJUNGVALL, I.; MICHAL, J. et al. Congenital heart defects in cats: a retrospective study of 162 cats (1996 e 2013). J. Vet. Cardiol., v.17, s215-s219, 2015.

WARE, W.A. Doenças cardíacas congênitas. In: (Eds.) Medicina interna de pequenos animais. 5 ed. Rio de Janeiro: Elsevier, 2015. p.96-114.

WESS, G.; KELLER, L.J.; KLAUSNITZER, M. et al. Comparison of longitudinal myocardial tissue velocity, strain, and strain rate measured by two-dimensional speckle tracking and by color tissue Doppler imaging in healthy dogs. $J$. Vet. Cardiol., v.13, p.31-43, 2011.

ZAKY, A.; DEEM, S.; BENDJELID, K.; TREGGIARI, M.M. Characterization of cardiac dysfunction in sepsis: an ongoing challenge. Shock, v.41, p.12-24, 2014. 